\title{
Slope Deformation Prediction Based on Support Vector Machine
}

\author{
Lei JIA ${ }^{1,2, *}$, Yuan $\mathrm{LI}^{2}$ and Yongping $\mathrm{XIE}^{2}$ \\ ${ }^{1}$ Dept. of Civil Engineering, Lanzhou Jiaotong University, Lanzhou, 730070-China \\ ${ }^{2}$ Dept. of exploration technology and engineering, Shijiazhuang University of Economics, Shijiazhuang,050031-China
}

Received 15 May 2013; Accepted 25 July 2013

\begin{abstract}
This paper principally studies the prediction of slope deformation based on Support Vector Machine (SVM). In the prediction process, explore how to reconstruct the phase space. The geological body's displacement data obtained from chaotic time series are used as SVM's training samples. Slope displacement caused by multivariable coupling is predicted by means of single variable. Results show that this model is of high fitting accuracy and generalization, and provides reference for deformation prediction in slope engineering.
\end{abstract}

Keywords: Deformation Prediction, Chaotic Time Series, Phase-space Reconstruction, Average Mutual Information Method

\section{Introduction}

In slope engineering, a series of displacement or stress physical quantities need to be monitored, but only a limited number of monitored data are available. The monitored data can be applied to analyze the evolution law of the geological body, predict its development trends and assess the engineering stability. Displacement and stress change of the slope engineering are subjected to many factors such as geological structure, lithology, hydrometeorology and engineering construction, hence the characteristics of randomness and fuzziness. The evolution of forcedeformation in geotechnical engineering is a typical nonlinear problem. The evolution process, highly nonlinear and complex, can hardly be described with simple mathematical or mechanical models.

The basic principle of the chaotic time series prediction is that the evolution of any component in the system is determined by other components interacted with it, and the information of these relevant components is implicit in the development process of any component. This law can be expressed as trajectories in high-dimensional space[1], [2],[3]. The theory of SVM(support vector machine) is classification and regression method developed on the basis of statistical theory. Support vector machine improves the generalization capability through the minimization of structural risk, providing a better solution to practical problems such as the small sample size, nonlinearity, high dimension and local minima[4], [5].

The deformation prediction of slope geological bodies is a complex system which is uncertain and nonlinear. Traditional methods can hardly describe these complex nonlinear characteristics in an accurate way and therefore can not accurately predict the deformation [6],[7],[8]. This paper conducted a single variable analysis on the multifactor coupling caused problems in slope engineering, and made nonlinear prediction on the displacement monitoring problems.

\section{Principles of SVM}

The principle of SVM is that through a pre-selected nonlinear mapping, the linear inseparable space is converted into a high-dimensional linear separable space, and in the feature space, optimal classification surface can be determined by the structural risk minimization principle. And nonlinear transformation can greatly simplify the calculation by defining the appropriate kernel function and converting the calculation of the complex nonlinear transformation into the calculation of dot product problems of the nonlinear transformation [9].

SVM has been successfully applied to the classification, curve fitting etc.. SVM applied in time series analysis is mainly related to the function fitting algorithm. For function fitting problems, the basic idea:On condition that the training sample $T=\left\{\left(x_{1}, y_{1}\right), \cdots,\left(x_{i}, y_{i}\right)\right\}, i=1, \cdots, n$, the estimated function $f(x)$ of the nonlinear problem can be described as: each sample point is mapped to a higher dimensional space by nonlinear mapping, and in high-dimensional space linear regression process is performed on mapping points, so that the original space nonlinear problem can be resolved.

The estimated function $f(x)$ can be expressed as:

$f(x)=\omega^{T} \cdot \varphi(x)+b$

\footnotetext{
*E-mail address: jialei1978@126.com

ISSN: 1791-2377 @ 2013 Kavala Institute of Technology. All rights reserved.
} 
Wherein: $\omega \in R^{n}$ is the weight value; $x \in R^{n}$ is input samples; $b \in R$ is deviation.

The purpose is to minimize Formula (2) by training $c$ and $\boldsymbol{b}$.

$R_{\text {reg }}(f)=C \sum_{i=1}^{n} \Gamma f\left(\left(x_{i}\right)-y_{i}\right)+\frac{1}{2}\|\omega\|^{2}$

Wherein: $C \sum_{i=1}^{n} \Gamma f\left(\left(x_{i}\right)-y_{i}\right)$ reflects the empirical risk; $\frac{1}{2}\|\omega\|^{2}$ is confidence interval which reflects the expressive power of function sets; $\Gamma(\cdot)$ is cost function; $C_{\text {is penalty }}$ function. $\boldsymbol{\omega}$ can be expressed with sample data as follows:

$\boldsymbol{\omega}=\sum_{i=1}^{n}\left(a_{i}-a_{i}^{*}\right) \varphi\left(x_{i}\right)$

Substitute equation (3) into equation (1), and new estimation function can be obtained:

$f(x)=\sum_{i=1}^{n}\left(a_{i}^{*}-a_{i}\right) K\left(x, x_{i}\right)+b$

$K\left(x, x_{i}\right)$ The purpose of the introduction of kernel function is to process the inner product computation in the high-dimensional space with simple function in lowdimensional space. Different kernel function will produce different support vector machines. Gaussian radial basis, polynomial and Sigmoid are commonly used kernel functions[9],[10]. indicates that $\mathrm{RBF}$ (Radical Basis Function) is the relatively common kernel function, therefore this article selects RBF as SVM kernel function.

\section{Chaotic time series model based on SVM}

Chaotic time series prediction is based on phase space reconstruction theory.For single variable time series, Packard et al. puts forward a method of reconstructing phase space[11].

to a given chaotic time series $x(t), t=1,2, \cdots, N$,a multi-dimensional phase space can be constructed from onedimensional time series by employing a certain time lag $\tau$ and embedding dimension $m$. Phase space vector constructed is:

$$
\boldsymbol{X}(t)=[x(t), x(t+\tau), \cdots, x(t+(m-1) \tau)]^{T}
$$

After the reconstruction of the phase space, the training of SVM can be carried out with the new data set obtained from the phase space reconstruction. The first step forecasting model of SVM at moment is as follows:

$$
\hat{x}_{t+1}=\sum_{i=1}^{N-(m-1) \tau}\left(a_{i}-a_{i}^{*}\right) \mathrm{K}\left(x_{i}, x_{i_{t}}\right)+b
$$

$$
\text { Wherein, } x_{i_{t}}=(x(t), x(t+\tau), x(t+2 \tau), \cdots, x(t+(m-1) \tau)) \text {, }
$$

Have $x_{t}=x(t)$, for the $t+1$ point of the phase space reconstruction:

$x_{i_{t+1}}=\left(\hat{x}_{t+1}, x(t+\tau), x(t+2 \tau), \cdots, x(t+(m-2) \tau)\right)$

Then prediction at the $t+2$ point can be obtained by the formula:

$\hat{x}_{t+2}=\sum_{i=1}^{N-(m-1) \tau}\left(a_{i}-a_{i}^{*}\right) \mathrm{K}\left(x_{i}, x_{i_{t+1}}\right)+b$

So the s-step SVM-based time series prediction model can be obtained accordingly:

$\hat{x}_{t+s}=\sum_{i=1}^{N-(m-1) \tau}\left(a_{i}-a_{i}^{*}\right) \mathrm{K}\left(x_{i}, x_{i_{t+s-1}}\right)+b$

In the above formula, $S$ is the predicted step parameter,

$x_{i_{t+s-1}}=\left(\hat{x}_{t+s-1}, \cdots, \hat{x}_{t+1}, \cdots, x(t+(m-s+2) \tau)\right)$

\section{The Application of SVM-based Chaotic time series model in Prediction of Slope safety}

\subsection{Basic information of the project}

The slope lithology of the permanent ship lock of the Three Gorges is mainly amphibole plagioclase granite. The fresh rock mass is hard and compact and is of good integrity; The thick surface of the weathering crust can be divided into completely weathered, strongly weathered, moderately weathered and weak weathered rock mass from top to bottom. Poor tectonite cementation and relatively developed structural plane and joint fissures of part of the rock mass are detrimental to slope stability. The location of cross section 19-19 has the highest slope, relatively poor rock mass integrity and complex geological conditions. Twelve measuring points of horizontal and vertical displacement are arranged in this cross-section. In this research, measuring point TP-BM10GP01 is selected as the research object. Samples of chaotic time series are established with the displacement monitoring data from 1995.6 1998.5, and dynamic displacement are monitored and predicted. The monitoring data are listed in Table1.

Table 1 Displacement observation data of the measuring point TPBM10GP01

\begin{tabular}{cccccc}
\hline Date & $\begin{array}{c}\text { Displace } \\
\text { ment } \\
/ \mathrm{mm}\end{array}$ & Date & $\begin{array}{c}\text { Displace } \\
\text { ment } \\
/ \mathrm{mm}\end{array}$ & Date & $\begin{array}{c}\text { Displace } \\
\text { ment } \\
/ \mathrm{mm}\end{array}$ \\
\hline 1995.6 & 0.00 & 1996.6 & 13.51 & 1997.6 & 18.62 \\
1995.7 & 0.97 & 1996.7 & 13.08 & 1997.7 & 18.37 \\
1995.8 & 3.35 & 1996.8 & 13.61 & 1997.8 & 18.78 \\
1995.9 & 4.77 & 1996.9 & 13.83 & 1997.9 & 18.65 \\
1995.10 & 6.17 & 1996.10 & 13.63 & 1997.10 & 19.13 \\
1995.11 & 9.50 & 1996.11 & 14.37 & 1997.11 & 19.90 \\
1995.12 & 12.04 & 1996.12 & 15.99 & 1997.12 & 21.47 \\
1996.1 & 13.24 & 1997.1 & 17.55 & 1998.1 & 22.17 \\
1996.2 & 12.67 & 1997.2 & 17.24 & 1998.2 & 22.32 \\
1996.3 & 13.70 & 1997.3 & 17.74 & 1998.3 & 21.87 \\
1996.4 & 13.74 & 1997.4 & 17.84 & 1998.4 & 22.60 \\
1996.5 & 13.17 & 1997.5 & 17.89 & 1998.5 & 23.56 \\
\hline
\end{tabular}




\subsection{Determination of Time Delay and Embedding Dimension}

In the process of reconstructing phase space, the selection of the appropriate time delay and embedding dimension is the key to phase space reconstruction. Whether the strange attractors' feature can be accurately described depends largely on the their accuracy.

\subsubsection{Average mutual information method to determine the delay time}

For the slope measured displacement time series $\left\{x_{n}\right\}$, the average mutual information between the actually measured values at time points $\tau$ and $n+\tau$ :

$$
I(\tau)=\sum_{n=1}^{N} P\left(x_{n}, x_{n+\tau}\right) \log _{2}\left[\frac{P\left(x_{n}, x_{n+\tau}\right)}{P\left(x_{n}\right) P\left(x_{n+\tau}\right)}\right]
$$

Wherein, $P\left(x_{n}\right), P\left(x_{n+\tau}\right), P\left(x_{n}, x_{n+\tau}\right)$ is probability.

Probability can be obtained by one-dimensional and twodimensional histograms. Generally the $\tau$ when $I(\tau)$ is at the first local minimum is selected as the delay time interval [12], [13].

\subsubsection{The False Adjacent Points Method to determine the embedding dimension}

The false adjacent points method is a method easier to achieve from the geometric point of view. According to this method, when the embedding dimension varies from $m$ to $m+1$, examine the adjacent points of the trajectory $X_{i}$ to differentiate real adjacent points from false ones. When there is no false adjacent points, the geometric structure is considered to be completely open[14], [15].

On condition that $X_{\eta(n)}$ is the nearest adjacent point of $X_{i}$, the distance between them is $\left\|X_{\eta(n)}-X_{i}\right\|^{(m)}$. When the dimension increases to $m+1$, the distance between them is $\left\|X_{\eta(n)}-X_{i}\right\|^{(m+1)}$. If $\left\|X_{\eta(n)}-X_{i}\right\|^{(m+1)}$ is much larger than $\left\|X_{\eta(n)}-X_{i}\right\|^{(m)}$ points of high-dimensional attractors, when projected onto low-dimensional trajectories, become adjacent points. Therefore such adjacent points are false ones.

To actually measure time series, have $m$ increase from 2 , and calculate the ratio of the false nearest adjacent points when the values of $m$ vary each time. The geometric structure of attractors is considered to be completely open till the ratio is less than $5 \%$ or the false nearest adjacent points no longer decrease with the increase of $m, m$ at this time is embedding dimension.

\subsection{Formation of SVM learning samples}

With MATLAB program and based on the measured displacement data, embedding dimension can be determined $m=4$ and delay time $\tau=3$, the reconstructed phase space constitutes training sample set in the form as follows:

$$
\begin{aligned}
& \boldsymbol{X}=\left[\begin{array}{cccc}
x_{1} & x_{1+\tau} & \cdots & x_{1+(m-1) \tau} \\
x_{2} & x_{2+\tau} & \cdots & x_{2+(m-1) \tau} \\
\vdots & \vdots & & \vdots \\
x_{n-(m-1) \tau} & x_{n-(m-1) \tau+1} & \cdots & x_{n-1}
\end{array}\right] \\
& \boldsymbol{Y}=\left[\begin{array}{c}
x_{2+(m-1) \tau} \\
x_{3+(m-1) \tau} \\
\vdots \\
x_{n}
\end{array}\right]
\end{aligned}
$$

Normalize training sample set in $[0,1]$. In this study, $70 \%$ of the total sample data are selected for training set, $20 \%$ for the test set, and $10 \%$ for the detection set. The training set is used to determine the SVM model, the test set to test the predictive capability of the model, and the detection set to test the model's outreach capacity. SVM's Kernel function adopts the Gaussian Radial Basis Function (RBF):

$\mathrm{K}\left(x, x_{i}\right)=\exp \left(-\frac{\left\|x-x^{i}\right\|^{2}}{\sigma^{2}}\right)$

For the values of $C$ and $\sigma$, the range of the parameter values shall be relatively wide. Set a larger step size to conduct circular trial calculation, and assess the various SVM training results obtained, thus the best SVM predicative model and its corresponding value of the parameter can be determined. Then set small step size to conduct circular trial calculation for values till the best SVM model and parameter values are determined[16]. The prediction result can be assessed with mean absolute error MAE. The formula for MAE is:

$M A E=\frac{1}{n} \sum_{i=1}^{n}\left|y_{i}-\hat{y}_{i}\right|$

Wherein: $\hat{y}_{i}$ is predictive value.

Carry out function fitting and prediction with the established SVM. The results and deviation analysis are shown in Table 2 and Figure 1.

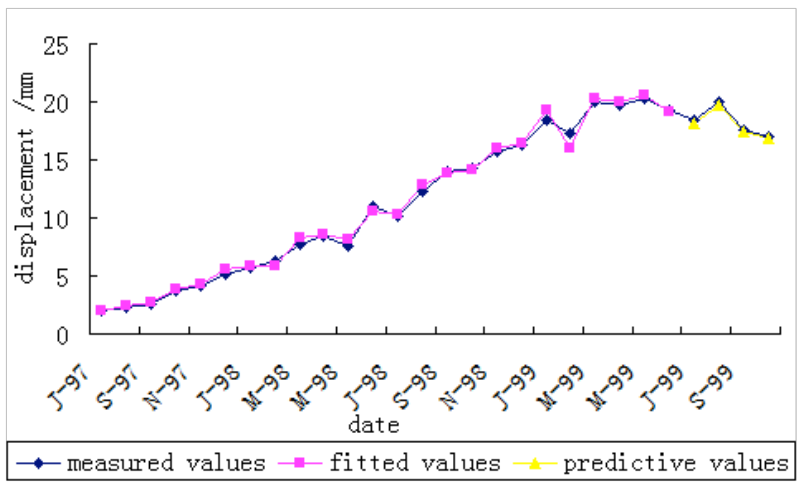

Fig 1. Comparison Chart of the displacement's fitted values, predictive values and measured values 
Table 2 Fitted values, predictive values and its mean absolute error

\begin{tabular}{ccccc}
\hline date & $\begin{array}{c}\text { displacement } / \\
(\mathrm{mm})\end{array}$ & $\begin{array}{c}\text { fitted values } \\
/(\mathrm{mm})\end{array}$ & $\begin{array}{c}\text { Predictive } \\
\text { values } \\
\text { / }(\mathrm{mm})\end{array}$ & $\begin{array}{c}\text { MAE } \\
/ \mathrm{mm})\end{array}$ \\
\hline $97-7$ & 1.94 & 2.040 & \\
$97-8$ & 2.32 & 2.420 & \\
$97-9$ & 2.53 & 2.760 & \\
$97-10$ & 3.72 & 3.820 & \\
$97-11$ & 4.18 & 4.283 & \\
$97-12$ & 5.18 & 5.590 & \\
$98-1$ & 5.71 & 5.810 & \\
$98-2$ & 6.26 & 5.920 & \\
$98-3$ & 7.73 & 8.270 & \\
$98-4$ & 8.43 & 8.529 & \\
$98-5$ & 7.54 & 8.190 & \\
$98-6$ & 10.99 & 10.540 & \\
$98-7$ & 10.16 & 10.260 & \\
$98-8$ & 12.33 & 12.810 & \\
$98-9$ & 14 & 13.900 & \\
$98-10$ & 14.24 & 14.110 & \\
$98-11$ & 15.66 & 16.050 & \\
$98-12$ & 16.32 & 16.420 & \\
$99-1$ & 18.5 & 19.300 & \\
$99-2$ & 17.26 & 16.050 & \\
$99-3$ & 19.98 & 20.350 & \\
$99-4$ & 19.71 & 20.030 & \\
$99-5$ & 20.27 & 20.590 & \\
$99-6$ & 19.22 & 19.121 & \\
$99-7$ & 18.43 & & \\
$99-8$ & 20.05 & & \\
$99-9$ & 17.58 & & \\
$99-10$ & 17.02 & & \\
\hline
\end{tabular}

As can be seen from Figure 1 and Table 2:

(1) displacement gradually increases over time and decreases after the peak, which not only reflects the displacement change of the surrounding rock with the loading and unloading in excavation, shoring and other constructions, but also indicates that the safety of the slope is stabilized
(2) The fitted values and measured values are better fitted with smaller errors if slope deformation is predicted after the reconstruction of phase space by using chaotic time series. The prediction is more accurate, and the mean absolute error of predictive values is only $0.618 \mathrm{~mm}$, which reflects the model's generalized epitaxial ability is superior.

\section{Conclusion}

(1) SVM is an approximate method of achieving structural risk minimization criterion. When the training samples are limited, SVM can provide good generalization ability and will not fall into the local minimum. In this paper, SVM regression principle, combined with the theory of delay coordinate reconstruction of phase space in chaotic dynamical system, helps establish a SVM prediction model of chaotic time series of slope displacement. The predictive results indicate that SVM-based prediction model is one with high accuracy and generalization in the prediction of slope deformation.

(2)Slopes engineering deals with complex natural geological body, and is affected by geological conditions, construction conditions and many other factors. The deformation evolution of slopes is a typical nonlinear, uncertain problem. This paper constructed training samples through chaotic time series, predicted the deformation of slopes by making use of SVM's regression characteristics and achieved the purpose of predicting slope displacement with high accuracy. The model can not only predict nonlinear time series of slope engineering, but also provide reference for many other fields of geotechnical engineering.

\section{Acknowledgment}

This work is supported by Science and technology research project of institutions of higher learning, Hebei Education Department, China (Fund No: Z2011224).

\section{References}

1. Casdagli M, "Nonlinear prediction of chaotic time-series", Physica D, May 1989,pp.335-356.

2. Han Liu, Ding Liu, Qi Li, "Chaotic Time Series Nonlinear Prediction Based on Support Vector Machines", System Engineering Theory and Practice, 2005 (9),pp.94- 99. (in Chinese)

3. Yixiang Fu, Zhiqiang Liu, "Analytic method and application about chaotic slope deformation destruction time series", Journal of Wuhan University of Technology (Transportation Science \& Engineering) 27(3), 2003, 473 -477. (in Chinese)

4. Hongbo Zhao, Xiating Feng, "Application of Support Vector Machines Function Fitting in Slope Stability Evalution", Chinese Journal of Rock Mechanics and Engineering 24(4), 2003, pp.241245. (in Chinese)

5. Haoxiang He, Weiming Yan, Lingyun Peng, "Damage Identification of Reinforced Concrete Bridge Based on Support Vector Machine",Journal of Highway and Transportation Research and Development 25 (3) , 2008,pp. 65- 69+89. (in Chinese)

6. Jingtai Niu, "Safety Monitoring Model Based on Displacement Mutation of High Slope", South-to-North Water Transfers and Water Science \& Technology 10(5), 2012, pp.84-87. (in Chinese)

7. Longjun Dong, Feiyue Wang, "Comprehensive Evaluation on Seismic Stability of Slopes Based on Unascertained Measurement", The Chinese Journal of Geological Hazard and Control 18(4), 2007, pp. 74-78. (in Chinese)

8. Xiaolong Tan, Weiya Xu, Guilan liang, "Research on Nonlinear Time Series Evolution Statistic Model of High Slope Displacements", Rock and Soil Mechanics 31(5) , 2010,pp. 1633 1 650. (in Chinese)
9. Xiuzhen Li, Jiming Kong, Chaofeng Li, "Application of Multiclassification Support Vector Machine in Regionalization of Debris Flow Hazards", Bulletin of Soil and Water Conservation 30(5) ,2010,pp.128-133. (in Chinese)

10. Guilan Liang, Weiya $\mathrm{Xu}$, Yuzhi He, "Study and application of PSO-RBFNN model to nonlinear time series forecasting for geotechnical engineering", Rock and Soil Mechanics 29(2), 2008,pp. 995-1 000. (in Chinese)

11. Hui Dong, Helin Fu, Wuming Leng, “ Kernel design for displacement time series of landslide",Rock and Soil Mechanics 29(4), 2008,pp.1 087-1 092. (in Chinese)

12. Hongguang Ma, "Selection of Embedding Dimension and Delay Time in Phase Space Reconstruction",Journal of Xi'an Jiaotong University 38(4), 2004,pp.335-338. (in Chinese)

13. TRELEA I $\mathrm{C}$, "The particle swarm optimization algorithm convergence analysis and parameter selection",Information Processing Letters, 2003, (85),pp.317-325.

14. Lansong Deng, Fei Shen, "Analysis and predication of fund index of nonlinear time series", Journal of Tianjin University 37(11) ,2004,pp.1 022-1 025.(in Chinese)

15. Hongzhi An, Min Chen, "Nonlinear Time Series Analysis", Shanghai:Shanghai Scientific and Technical Publishers, 1998.pp.127-129.(in Chinese)

16. Chuangbing ZHou, Yifeng Chen, "Application of Phase Space Reconstruction in Slope Displacement Forecasting", Rock and Soil Mechanics 21(3), 2000,pp. 205-208. (in Chinese) 